\title{
aDolescents gEnder surVey, rEsponsible coupLes evaluation, and capacity building Project in India (DEVELOP): a study
} protocol [version 1; peer review: 2 approved with

\section{reservations]}

\author{
Anand Ahankari (iD1, Mark Hayter1', Clare Whitfield (iD1, Parveen Ali2, \\ Sneha Giridhari3 ${ }^{3}$, Shruti Tambe ${ }^{4}$, Pratyush Kabra5 , Kranti Rayamane6, \\ Pavel Ovseiko (iD) 7
}

\footnotetext{
${ }^{1}$ School of Health and Social Work, Faculty of Health Sciences, University of Hull, Hull, Yorkshire, HU6 7RX, UK

${ }^{2}$ School of Nursing and Midwifery, University of Sheffield, Sheffield, S10 2LA, UK

${ }^{3}$ SWISSAID, Pune, Maharashtra, 411040, India

${ }^{4}$ Department of Sociology, Savitribai Phule Pune University, Pune, Maharashtra, 411007, India

${ }^{5}$ Department of Community Medicine, Ashwini Rural Medical College, Hospital and Research Centre, Solapur, Maharashtra, 413006, India

${ }^{6}$ Halo Medical Foundation, Andur, Osmanabad, Maharashtra, 413603, India

${ }^{7}$ Radcliffe Department of Medicine, University of Oxford, Oxford, OX3 9DU, UK
}

V1 First published: 24 Jun 2019, 8:958

https://doi.org/10.12688/f1000research.19521.1

Latest published: 29 Mar 2021, 8:958

https://doi.org/10.12688/f1000research.19521.2

\section{Open Peer Review}

Approval Status

\section{1}

2

version 2

(revision)

29 Mar 2021

version 1

24 Jun 2019

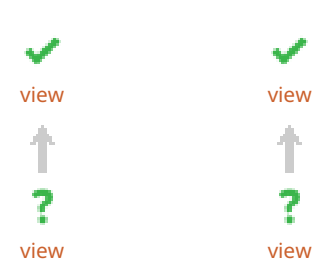

1. Prashanth N Srinivas (iD), Institute of Public

Health, Bengaluru, India

\section{Katharina Goessmann (iD), Bielefeld \\ University, Bielefeld, Germany}

Any reports and responses or comments on the article can be found at the end of the article. 
intervene during violence, and to provide support services for women and their family members. Research findings will be disseminated though public engagement events in India, international conferences, and peer reviewed publications. This will impact on the policy and work models of Indian partners to plan future project investments. Research findings will be also useful for local government authorities and

non-government agencies striving to advance gender equality.

\section{Keywords}

Gender, Maharashtra, India, Adolescent, Violence, Evaluation

Corresponding authors: Anand Ahankari (dr.anandahankari@gmail.com), Mark Hayter (M.Hayter@hull.ac.uk)

Author roles: Ahankari A: Conceptualization, Funding Acquisition, Methodology, Project Administration, Writing - Original Draft Preparation, Writing - Review \& Editing; Hayter M: Conceptualization, Funding Acquisition, Methodology, Supervision, Writing - Review \& Editing; Whitfield C: Methodology, Writing - Review \& Editing; Ali P: Methodology, Writing - Review \& Editing; Giridhari S: Funding Acquisition, Methodology, Supervision, Writing - Review \& Editing; Tambe S: Methodology, Writing - Review \& Editing; Kabra P: Writing - Review \& Editing; Rayamane K: Writing - Review \& Editing; Ovseiko P: Methodology, Writing - Original Draft Preparation, Writing Review \& Editing

Competing interests: DEVELOP project has an evaluation research component (section II. Qualitative component) focusing on the ongoing gender equality programme, which is funded by the SWISSAID. SWISSAID is a lead partner in India to support DEVELOP research activities. Ms Sneha Giridhari, who is involved in this project and also one of the authors, is currently employed by the SWISSAID as a programme officer and is involved in monitoring the DEVELOP project progress.

Grant information: The DEVELOP project is formulated following a travel grant awarded to Prof Mark Hayter and Dr Anand Ahankari in May 2018 from the Global Challenges Research Fund (GCRF) allocated to the University of Hull, UK. The project received a dedicated grant in November 2018 from the Global Challenges Research Fund (GCRF) allocated to the University of Hull, UK. Dr Pavel Ovseiko is supported by the European Union's Horizon 2020 research and innovation programme award STARBIOS2 [709517] and by the National Institute for Health Research, grant NIHR Oxford Biomedical Research Centre [BRC-1215-20008] to the Oxford University Hospitals NHS Foundation Trust and the University of Oxford.

The funders had no role in study design, data collection and analysis, decision to publish, or preparation of the manuscript.

Copyright: $\odot 2019$ Ahankari A et al. This is an open access article distributed under the terms of the Creative Commons Attribution License, which permits unrestricted use, distribution, and reproduction in any medium, provided the original work is properly cited.

How to cite this article: Ahankari $A$, Hayter $M$, Whitfield $C$ et al. aDolescents gEnder surVey, rEsponsible coupLes evaluation, and capacity building Project in India (DEVELOP): a study protocol [version 1; peer review: 2 approved with reservations] F1000Research 2019, 8:958 https://doi.org/10.12688/f1000research.19521.1

First published: 24 Jun 2019, 8:958 https://doi.org/10.12688/f1000research.19521.1 


\section{Introduction}

Gender-based violence against women is a fundamental violation of women's human rights, health, and wellbeing ${ }^{1}$. Globally, intimate partner violence (IPV) is the most common form of gender-based violence against women ${ }^{1}$. A recent study from south India reported that over $50 \%$ of women had experienced physical domestic violence ever, and $27 \%$ faced physical domestic violence in the past six months ${ }^{2}$. The majority of female victims are married to the perpetrator and underreporting of IPV is a known phenomenon $^{3,4}$. IPV is linked to a range of factors including alcohol addiction, financial debt, cultural and social acceptance of violence, and childhood trauma/exposure to violence ${ }^{4}$. These factors are also linked to gender-based discrimination, adversely affecting girls' and women's health and well-being, their ability to continue their education, choose a career, make informed reproductive decisions, and achieve financial independence. Adverse effects of gender-based discrimination on girls' and women's health and wellbeing are particularly high in deprived communities $^{3,4}$.

Although the need to reduce gender-based violence and empower women in India is widely recognised, there is very limited information available on gender equality-related knowledge, attitudes, and behaviours among Indian adolescents, who are an important age group to target with public health and development interventions. Moreover, traditionally, interventions to reduce gender-based violence and empower women were focused on women, and men were predominantly left out of such interventions. Overall, there is very limited research on the effectiveness of gender-based violence reduction interventions.

With financial and technical support from the SWISSAID ${ }^{5}$, Halo Medical Foundation (HMF), an NGO working in Maharashtra state of India, has developed and is currently implementing the 'Responsible Couples' intervention to address gender-based violence against women by educating men, supporting women, and providing village infrastructural facilities to create healthy relationships and violence-free communities ${ }^{6}$. The 'DEVELOP' project seeks to increase understanding of gender equality-related knowledge, attitudes, and behaviours among Indian adolescents, as well as evaluate the 'Responsible Couples' intervention. The project is planned to be conducted in Maharashtra state of India in 2019. This paper is a study protocol of the DEVELOP providing detailed information on study objectives, research methods of two main components of the project, data storage, handling, and dissemination plans.

\section{Protocol}

Project goal

To conduct feasibility and capacity building work in India to support future research and development projects in gender equality.

\section{Research objectives}

- To inform the development of a survey tool measuring gender equality related knowledge, attitudes, and behaviours in Indian adolescents.

- To conduct a qualitative evaluation of the current genderbased violence reduction intervention-the 'Responsible
Couples' project in the rural region of Maharashtra state of India.

\section{Study design}

This study has two components as outlined below to achieve the research objectives. The project team structure and partners are outlined in Figure 1.

I. Quantitative component - a questionnaire survey of the gender equality beliefs and attitudes of minimum 1000 young people (male and female) aged 16 to 19 from 70 villages of Maharashtra state, India.

II. Qualitative component - focus group discussions with the local gender equality promotion groups in 12 villages of Maharashtra state to explore their views on the implementation of the 'Responsible Couples' project, what challenges they are experiencing in their gender equality work, what they feel about the impact of their work, and how the project can be improved further.

\section{Quantitative component}

\section{Selection and recruitment}

The project field consists of 70 villages from HMF's area of work, located in Osmanabad district of Maharashtra state of India. The study will invite all adolescents aged 16 to 19 from 70 villages to participate in the project by completing a questionnaire. These villages define HMF's current geographic scope based on funding allocated by the SWISSAID to work in Maharashtra. Future programme and intervention development work will involve the same villages; therefore, these were considered in the DEVELOP research project.

Each village has a member of HMF staff linked to it. This individual will help distribute written information about the project (in the form of a leaflet, Supplementary File 1, Extended data ${ }^{7}$ ) in each village at least two weeks before any data is collected. Leaflets will be distributed to cover all community areas in each village. There are also field staff such as healthcare workers in project area. These workers are also able to provide any verbal description of the project if required at this point in time. They will also orally inform of the date of the data collection event. All staff members including field staff received necessary research and ethics training in February and April 2019 and are supervised by a senior research co-ordinator on a daily basis with additional support from a project manager based at HMF.

Data collectors will then visit the village for one day of data collection. They will set up their station at a village health centre, school or other locally available building/resource, where interested adolescents will be invited to visit to find out more about the project. Written and verbal explanations will be given to those who have not seen the previous information sheet (Supplementary 1, Extended data $)^{7}$.

The study will be open to boys and girls aged 16 to 19 only. Participants should be able to read and write in local language (Marathi) in order to understand the project information sheet and complete the data collection form independently. Data collection documents 


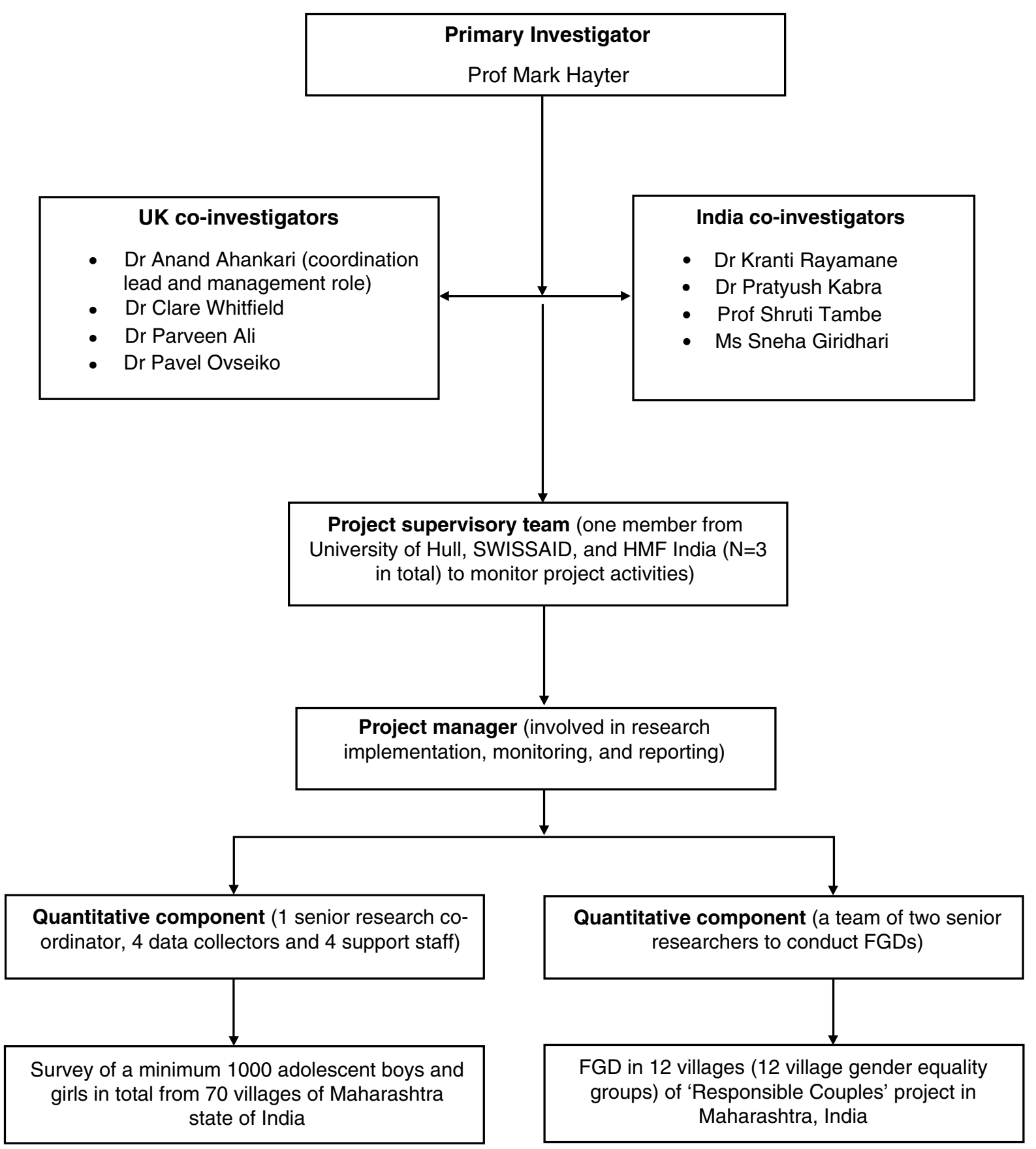

Figure 1. DEVELOP project structure. HMF, Halo Medical Foundation; FGD, focus group discussion.

were translated from English to Marathi by a project manager in the first instance, and the translation was verified by authors with bilingual proficiency (AA and SG). The final data files were reviewed several times to ensure its accuracy. The adolescents who agree to participate will be given a questionnaire, pen, and sealable envelope. The on-site data collection staff will address any queries, if asked by participants.

\section{Sample size}

The adolescent survey will be conducted to collect a minimum of 1000 questionnaires. This number is based on discussions with project partners to ensure that results from this study will be applicable to rural areas of Maharashtra state. This is also the maximum number of participants this project could achieve considering available resources. A similar 
strategy was used to conduct research in this field area involving adolescent girls ${ }^{8}$.

In order to collect representative data by age and gender, a stratified sampling technique will be used. In total, a minimum 1000 questionnaires will be completed, including 125 from each age group ${ }^{16,17,18,19}$ and 500 from each gender. This is the ideal sample scenario; however, no participants will be turned away on a data collection day even if the said number has already been achieved.

\section{Survey instrument}

A survey questionnaire in the local language (Marathi) will be used to collect data from adolescents. An English version of the questionnaire is available as Extended data ${ }^{7}$. The tool is developed based on a validated and published questionnaire ${ }^{9,10}$, which was used to study gender equality among Indian adolescents ${ }^{9}$. The questionnaire was iteratively revised and improved within the team, discussed with partners, and then piloted and validated prior to administration. The outlined process was completed through focus group discussions and a testing phase involving adolescent boys and girls at the HMF training centre in March 2019. The feedback from the discussions was included, and minor changes were made mainly on the structure of the data collection form. No major changes such as question re-structuring were required. The questionnaire used in this study has a section on basic demographics (12 questions) and then three individual sections to measure knowledge (nine statements), attitudes (six statements) and behaviours (seven statements) related to gender equality (Supplementary 2, Extended data) ${ }^{7}$. The gender equality score for each participant will be calculated for the three sections of the questionnaire (knowledge, attitudes and behaviour) using the following method. For each statement, the score will range from zero to two. Those who agreed with a given statement, indicating a lack of support for gender inequality, receive a score of zero. Those who partially agree receive a score of one, and those who disagreed receive a score of two, indicating support for gender equality. The total score will be calculated for each completed questionnaire by adding the score for all 22 statements. Total scores for each questionnaire will range from a low of zero (highly gender inequitable) to a high of 44 (highly gender equitable).

\section{Data collection, analysis and storage}

To ensure confidentiality, questionnaires will be completed in an area of the village hall/health centre that affords privacy. The completed questionnaires (in sealed envelopes) are then placed in a box by participants as they leave the hall/data collection centre. The overall data collection will be supervised by a qualified member of HMF staff.

The questionnaire will not collect any personal identifiable information such as name, home address, or contact details. Both the study information sheet and the questionnaire will include information assuring the participants of confidentiality and how the data will be used. Participants will not be identified or identifiable through reports or publications and only the research team will have access to the data. All data in India will be stored on a password-protected computers and encrypted USB devices and will only be accessible to the project and research teams. The survey data will be moved to the University of Hull, UK in a Microsoft Excel file, stored on a secure server and used for analysis purposes. A member of the research team will access the data stored at HMF office in person and will upload the data remotely to the University of Hull online storage server using secured login details. This will be verified by another team member to ensure that all data are safely moved to the University of Hull online storage system. The data will be stored for five years following the project completion.

Incomplete questionnaires will be discarded from analysis and stored for auditing purposes. Survey responses will be analysed in Stata (StataCorp, College Station, Texas, USA) and/or SPSS (IBM) using descriptive statistics, tests of statistical significance, and reliability coefficients. If data permits, then additional analysis to compare results across villages/blocks/districts would be also conducted. Results will be reported in line with STROBE guidelines $^{11}$.

\section{Qualitative component}

\section{Selection and recruitment}

The 'Responsible Couples' project is currently being implemented in 40 villages of Osmanabad district of Maharashtra state, India. Each village has one local group comprised of 15 to 20 village members, who are working towards gender equality in their community. Group members have been trained by subject experts and receive mentoring support from HMF project implementation staff. The groups provide support and facilitate access to victims of IPV and intervene to prevent violence against women, focussing on those who are married and living with their husband and/or in-laws. Importantly, as part of HMF's work to support research development, these groups have been involved in the inception of the current research study.

From a list of 40 villages, 12 villages will be randomly selected for focus group discussions. In order to ensure random selection, all villages will be randomly numbered by a project manager based in India, and a total of 12 numbers will be selected by a member of the research team (AA, based in the UK). The process will be completed over email to record the process. At least two weeks before focus group discussions are held, members of the research/ project team will convene meetings with the members of the village gender equality groups in the selected villages to describe the project and answer questions. To accompany the verbal description of the project, each group member will receive an information sheet (Supplementary 3, Extended data) ${ }^{7}$. Only existing members of the village gender equality groups in the selected villages will be invited to participate in focus group discussions.

\section{Sample size}

Twelve focus group discussions are expected to provide sufficient insights into the implementation of the 'Responsible Couples' intervention across 40 participating villages. It is expected that up to 10 members from each village will participate in each focus group discussion. Based on our series of consultations with partners, field visits, interactions with beneficiaries, the proposed 12 focus group discussions is expected to be sufficient to achieve data saturation. 


\section{Focus group discussion instrument}

A discussion guide in a local language (Marathi) will be used to facilitate focus group discussions. An English version is included as Extended data (Supplementary 4) ${ }^{7}$. Data collection documents were translated from English to Marathi by a project manager at first instance and were verified by authors with bilingual proficiency (AA and SG). The final data files were reviewed several times to ensure its accuracy. The discussion guide was revised iteratively within the team, discussed with partners, and then piloted prior to research use. The interview guide was used to conduct discussions in two villages from the project areas where its structure, questions were tested. This was attended by a project manager and a senior research co-ordinator to provide feedback to investigators based in India and the UK. No amendments to the guide were required.

\section{Data collection, analysis and storage}

Focus group discussions will be conducted, transcribed, and translated by two experienced facilitators with bilingual skills (Marathi and English) under the supervision of the research team. The qualitative data will include information on the views of village level groups on: the implementation of the 'Responsible Couples' project; what challenges they are experiencing in their gender equality work; what they feel about the impact of their work; and how the project can be improved further.

No personal information will be collected during the focus groups. The information sheet will include assurances on confidentiality and that no identifiable data will be used in reports and publications. Only the research team will have access to the data. All data in India will be stored on password-protected computers and encrypted USB devices and will only be accessible to the research team. Once research activities are completed in India, the focus group discussion data will be moved to the University of Hull, UK in Microsoft Word and PDF files with the audio recorded discussion files, stored on a secure server and used for analysis purposes, as described for the quantitative data.

Qualitative data from the focus group discussions will be analysed thematically and organised in NVivo (QSR International). Two researchers will independently code data and synthesise the findings into themes. They will then meet to discuss areas of agreement and disagreement and reach consensus on the coding tree, illustrative quotations, and interpretation. Results will be reported in line with COREQ guidelines ${ }^{12}$.

\section{Ethical statement}

The study has been approved by the Faculty of Health Sciences Ethics Committee, University of Hull, UK (approval reference number- FHS125 issued on 3rd April 2019) and the ethics committee of the Ashwini Rural Medical College, Hospital and Research Centre, Solapur, Maharashtra, India (approval reference number- ARMCH/IECHR/03/2019 issued on 15th March 2019). All survey participants will give individual oral informed consent before completing the questionnaire. The oral informed consent was preferred in this survey to ensure full confidentiality of participating adolescents. Written consent requires basic details such as name, address with further requirements of anonymisation. These details are not requested on the questionnaire and therefore oral informed consent is deemed sufficient from a willing participant before handling over the questionnaire. As a result of this, no personal information on participating adolescents such as name or address are collected at any point of time. All focus group participants will give a collective written informed consent before participating in focus group discussions. This strategy was decided following consultation with our project partners. At the start of the session, a collective signed consent form will be obtained. In India, the provision of a personal signature on a form is regarded with some suspicion and the collective form alleviates this. Further, all eligible participants are active members of the village level group and regularly meet for monthly meeting and thus are aware about collective signatures as a part of on-going project activities. This also allows the data collection team to collect the signatures of willing participants where individual names, addresses and contact details were not collected. The consent form is provided as Extended data (Supplementary 5) ${ }^{7}$.

\section{Dissemination of information}

The results of the study will be disseminated via local, regional, and national dissemination events, online video and blogs, peer-reviewed publications, and presentations at international conferences.

\section{Study status}

The DEVELOP project duration is from December 2018 to July 2019. The study is currently in the data collection phase (planned to take place from April to July 2019).

\section{Conclusions}

To the best of our knowledge, this will be the first survey from the Maharashtra state of India, and one of the largest surveys, measuring gender equality-related knowledge, attitudes, and behaviours among Indian adolescents. The survey findings will generate new valuable insights into how adolescent groups could be engaged in the future to improve gender equality in Indian communities.

The qualitative evaluation will inform the implementation of the 'Responsible Couples' intervention and strategies to improve it. It will also have policy implications for HMF, SWISSAID, and other organisations seeking to reduce gender-based violence and empower girls and women in Maharashtra and other states of India. Considering diverse Indian culture, practices, and beliefs, the study results may not be completely generalizable beyond the population studied.

The DEVELOP project will contribute to research capacity building and evidence-based practice in a limited resource setting. The project will provide opportunities to train and engage over 10 researchers and practitioners in Maharashtra state of India to improve their knowledge, develop new research skills, and enhance their experience of collaborating with international partners. It is expected that the project will help partners involved from India and the UK to develop further research on the adoption, implementation, and scale-up of evidence-based gender equality 
interventions in Maharashtra and other Indian states and territories.

\section{Data availability}

Underlying data

No underlying data are associated with this article.

\section{Extended data}

Figshare: ADolescents GEnder SurVey, REsponsible CoupLes EvaluatiOn, and Capacity Building Project in India (DEVELOP): A study protocol. https://dx.doi.org/10.6084/m9.figshare.8256050. $\mathrm{V} 1^{7}$

This project contains the following extended data:

- Supplementary Files 1 to 5.pdf (participant information sheet for questionnaire, questionnaire in English, participant information sheet for focus group discussion, focus group discussion guide in English, focus group discussion consent form)

- DEVELOP_Survey questionnaire Marathi.pdf (questionnaire in Marathi)

- DEVLOP_FGD Guide Marathi.pdf (focus group discussion guide in Marathi)

Data are available under the terms of the Creative Commons Zero "No rights reserved" data waiver (CCO 1.0 Public domain dedication).

\section{Grant information}

The DEVELOP project is formulated following a travel grant awarded to Prof Mark Hayter and Dr Anand Ahankari in May 2018 from the Global Challenges Research Fund (GCRF) allocated to the University of Hull, UK. The project received a dedicated grant in November 2018 from the Global Challenges Research Fund (GCRF) allocated to the University of Hull, UK. Dr Pavel Ovseiko is supported by the European Union's Horizon 2020 research and innovation programme award STARBIOS2 [709517] and by the National Institute for Health Research, grant NIHR Oxford Biomedical Research Centre [BRC-1215-20008] to the Oxford University Hospitals NHS Foundation Trust and the University of Oxford.

The funders had no role in study design, data collection and analysis, decision to publish, or preparation of the manuscript.

\section{Acknowledgements}

Firstly, we thank the Research Facilitation Office of the University of Hull, UK for providing financial support through the Global Challenge Research Fund Pump Priming Grant initiative (GCRF Pump Priming Award 2018-19). We acknowledge the support received from the SWISSAID India liaison office, and Halo Medical Foundation, India for offering access to project area and sharing their experiences to conceptualise this study and also the grant application. We thank Dr Shashikant Ahankari (President, HMF) and Ms Kavita Gandhi (Country Representative, SWISSAID India) for their support and guidance. We also thank Mr Milind Hardikar (SWISSAID India) for his support in finance monitoring and project administration.
1. World Health Organisation: Violence against women. [accessed on 5 May 2019]. Reference Source

2. Rocca $\mathrm{CH}$, Rathod $\mathrm{S}$, Falle $\mathrm{T}$, et al:: Challenging assumptions about women's empowerment: social and economic resources and domestic violence among young married women in urban South India. Int J Epidemiol. 2009; 38(2): 577-585.

PubMed Abstract | Publisher Full Text | Free Full Text

3. Fulu E, Warner X, Miedema S, et al:: Why Do Some Men Use Violence Against Women and How Can We Prevent It? Quantitative Findings from the United Nations Multi-country Study on Men and Violence in Asia and the Pacific. Bangkok: UNDP, UNFPA, UN Women and UNV, 2013. Reference Source

4. International centre for research on women: Men, Masculinity andDomestic Violence in India. [accessed on 10 May 2019]. Reference Source

5. SWISSAID, India. [accessed on 6 June 2019]. Reference Source

6. Halo Medical Foundation, India. [accessed on 6 June 2019] Reference Source

7. Ahankari A, Hayter M, Whitfield C, et al:: ADolescents GEnder SurVey, REsponsible CoupLes EvaluatiOn, and Capacity Building Project in India
(DEVELOP): A study protocol. 2019

http://www.doi.org/10.6084/m9.figshare.8256050.v1

8. Ahankari AS, Myles PR, Fogarty AW, et al:: Prevalence of iron-deficiency anaemia and risk factors in 1010 adolescent girls from rural Maharashtra, India: a cross-sectional survey. Public Health. 2017; 142: 159-166. PubMed Abstract | Publisher Full Text

9. Achyut P, Bhatla N, Khandekar S, et al:: Building Support for Gender Equality among Young Adolescents in School: Findings from Mumbai, India. ICRW, New Delhi. [accessed on 16 May 2019]. Reference Source

10. Nanda G: Compendium of Gender Scales. Washington, DC: FHI 360/C-Change 2011. [accessed on 16 May 2019]. Reference Source

11. von Elm E, Altman DG, Egger M, et al:: The Strengthening the Reporting of Observational Studies in Epidemiology (STROBE) statement: guidelines for reporting observational studies. J Clin Epidemiol. 2008; 61(4): 344-9. PubMed Abstract | Publisher Full Text

12. Tong A, Sainsbury P, Craig J: Consolidated criteria for reporting qualitative research (COREQ): a 32-item checklist for interviews and focus groups. Int $J$ Qual Health Care. 2007; 19(6): 349-357. PubMed Abstract | Publisher Full Text 


\section{Open Peer Review}

\section{Current Peer Review Status: ? ?}

\section{Version 1}

Reviewer Report 26 February 2021

https://doi.org/10.5256/f1000research.21401.r77963

(C) 2021 Goessmann K. This is an open access peer review report distributed under the terms of the Creative Commons Attribution License, which permits unrestricted use, distribution, and reproduction in any medium, provided the original work is properly cited.

\section{Katharina Goessmann \\ Department of Psychology, Bielefeld University, Bielefeld, 100131, Germany}

The manuscript, entitled "aDolescents gEnder surVey, rEsponsible coupLes evaluation, and capacity building Project in India (DEVELOP): a study protocol", presents a study protocol of a proposed study to be conducted within a capacity building project (called DEVELOP) that seeks to plan and perform programs to address gender-based violence (GBV) in India, which is a serious issue with numerous negative consequences, as the authors outline in their introduction. The manuscript reports on two components of the project: a quantitative survey on genderrelated knowledge, attitudes and practices among adolescents to improve the evidence base in this regard, as well as qualitative evaluation of an ongoing GBV intervention ("Responsible Couples", RC) in Osmanabad district of Maharashtra, India.

In addition to those points raised by the first reviewer, I have a few remarks that might help to further improve the manuscript.

\section{Introduction:}

From the Title, Abstract and the first part of the introduction, it is not clear whether the study focuses on domestic/partner violence against women, or refers to any kind of GBV against women. Please clarify this from the beginning; also, in the summary of literature from studies reporting GBV globally and in India.

The authors state that existing intervention programs to reduce GBV have predominantly focused on women. I suggest adding some more information here on why it is important to include men/boys in those efforts.

It would also be interesting to learn a bit more about the background of the DEVELOP project already in the introduction, such as what are its expected advantages, whether there are other comparable approaches and so on.

\section{Quantitative component:}

Selection and recruitment: 
The envisaged number of participants in the quantitative survey is 1000 . What is the time frame in which you are planning to achieve this number?

How will the under-age participants give their informed consent? Is the informed consent of parents/guardians required here?

Survey questionnaire (Extended data): In my opinion, the wording of some items could be slightly changed in order to avoid misunderstandings and allow meaningful interpretation:

Part A, item 5: In its current wording, the item might be understood as asking for the status quo of what "a women's role" usually is in society or what the adolescents experience in their daily life. Like this, their answer to the item is not necessarily reflective of a lack of support for gender inequality, as it does not directly ask whether the respondent agrees with this widespread societal role of women, or if they think it should be like that. If the item aims to assess the adolescents' gender beliefs and attitudes, I suggest rephrasing it slightly, for example like this: A woman's role should be taking care of her house and family members / A real/good woman's role is taking care of her house and family members. Similarly, in item 8.

Part B, item 10: In this item to it is unclear whether it is asking for the adolescents' opinion/belief in this regard, or for their experience. This should be either rephrased or carefully considered in the interpretation of the data.

\section{Qualitative component:}

Data collection:

Given the sensitivity of the topic, the participants' gender identities should be considered. Will the focus groups consist of members of all genders or will they be separate for men and women members of the local gender promotion groups? Or are they all female anyway? And what about the gender of the research staff conducting the focus groups?

\section{Conclusions:}

The authors state that "the project will provide opportunities to train and engage over 10 researchers and practitioners in Maharashtra state of India to improve their knowledge, develop new research skills, and enhance their experience of collaborating with international partners". From the described procedures, this expected outcome is not apparent. Please elaborate on what this assumption/aim is based on.

\section{Minor corrections:}

Figure 1: the second lowest box on the right should read "Qualitative component"

Is the rationale for, and objectives of, the study clearly described?

Partly

Is the study design appropriate for the research question?

Yes

Are sufficient details of the methods provided to allow replication by others? 
Partly

Are the datasets clearly presented in a useable and accessible format?

Not applicable

Competing Interests: No competing interests were disclosed.

Reviewer Expertise: partner violence, violence against women, psychotraumatology

I confirm that I have read this submission and believe that I have an appropriate level of expertise to confirm that it is of an acceptable scientific standard, however I have significant reservations, as outlined above.

Author Response 11 Mar 2021

Anand Ahankari, University of Hull, Hull, UK

Response to comments from reviewer 2 (Katharina Goessmann)

1. The manuscript, entitled "aDolescents gEnder surVey, rEsponsible coupLes evaluation, and capacity building Project in India (DEVELOP): a study protocol“", presents a study protocol of a proposed study to be conducted within a capacity building project (called DEVELOP) that seeks to plan and perform programs to address gender-based violence (GBV) in India, which is a serious issue with numerous negative consequences, as the authors outline in their introduction. The manuscript reports on two components of the project: a quantitative survey on gender-related knowledge, attitudes and practices among adolescents to improve the evidence base in this regard, as well as qualitative evaluation of an ongoing GBV intervention ("Responsible Couples", RC) in Osmanabad district of Maharashtra, India.

In addition to those points raised by the first reviewer, I have a few remarks that might help to further improve the manuscript.

Response: Thank you for your careful reading of the manuscript and positive feedback. We believe your comments and suggestions have helped us to improve the quality of the manuscript. Please find below our point-by-point responses and revisions.

\section{Introduction:}

From the Title, Abstract and the first part of the introduction, it is not clear whether the study focuses on domestic/partner violence against women, or refers to any kind of GBV against women. Please clarify this from the beginning; also, in the summary of literature from studies reporting GBV globally and in India.

Response: We have now revised this across the study protocol by using two key termsDomestic Violence and Assault (DVA), where intimate partner violence (IPV) is the most common form. Revisions were made to ensure consistency across our writing.

3. The authors state that existing intervention programs to reduce GBV have predominantly focused on women. I suggest adding some more information here on why it is important to include men/boys in those efforts. 
Response: We have now expanded further on this section as follows.

Although the need to reduce DVA and empower women in India is widely recognised, there is very limited information available on gender equality-related knowledge, attitudes, and behaviours among Indian adolescents, who are an important age group to target with specific interventions. Interventions to reduce DVA were primarily focused on women for a long-time, and men were not involved in such intervention initiatives. This results in a lack of awareness in men who are often perpetrators subjecting risks to interventions targeted to reduce DVA. However, creating such awareness among men is challenging as it requires change in their attitudes, behaviours by empowering them with knowledge on the importance of gender equality and also on negative impacts of DVA on women and children. Development work to initiate such change requires challenging existing social norms, which come from centuries-old cultural practices, where discrimination against women/girls caused gender-based allocation of resources, work and opportunities across lifespan. Therefore, involvement of men in gender equality related work broadly aims to initiate and sustain a change at individual, family and community level to improve health and wellbeing of women. There is very limited research on the effectiveness of DVA reduction interventions especially from rural and difficult to access communities. In India, men engagement expanded in the recent decade thus evaluating such programmes are imperative to inform future work.

4. It would also be interesting to learn a bit more about the background of the DEVELOP project already in the introduction, such as what are its expected advantages, whether there are other comparable approaches and so on.

Response: Details of the conceptualisation of the DEVELOP project, its relevance and collaborations are explained at the start of the protocol prior to goals and objectives.

DEVELOP project is designed primarily to support new research collaborations by generating evidence mainly from rural areas of the Maharashtra state of India. The evidence generation is proposed by two key objectives which fit under the overarching project goal as explained below. Research work will involve local staff working full-time at HMF (NGO partner) who will be trained to develop their research capacities and will be engaged in data collection work. Research undertaken through both objectives by a local team will provide them 'real world' experiences supporting their future career development. Secondly, our two key partners (SWISSAID and HMF) will benefit from such evidence to inform their on-going as well as forthcoming projects on gender equality in India. The first objective will help our key partners plan projects involving young people through their future expansion, and the second objective will contribute to improve their on-going intervention approach. SWISSAID works with several Indian NGOs, thus findings will be useful in other areas of Maharashtra and nationally in India to design gender equality related work. Research findings, capacity building initiative and collaboration work will also offer valuable experiences for all partners involved to plan future initiatives. The proposed structure helped design the project in line with our funding requirements where local capacity building and research goals are incorporated into this model.

\section{Quantitative component:}


Selection and recruitment:

The envisaged number of participants in the quantitative survey is 1000 . What is the time frame in which you are planning to achieve this number?

Response: We have revised these details as mentioned earlier in this letter in response to the first reviewer and also addressed your question. The revision is outlined below.

The adolescent survey will be conducted to collect a minimum of 1000 questionnaires. This number is based on discussions with project partners to ensure that study is deliverable in given resources and time. Research findings may be applicable to wider areas of rural Maharashtra state. The proposed target of 1000 is set considering three months will be available to collect data, however if permitted higher sample size will be achieved depending on project progress. $A$ similar strategy was used to conduct research in this field area involving adolescent girls $\underline{8}$. In order to collect representative data by age and gender, a stratified sampling effort will be undertaken. In total, a minimum 1000 questionnaires will be completed with an aim to collect 125 from each age group $(16,17,18,19$ years), and 500 from each gender. This is the ideal sample scenario; however, no participants will be turned away on a data collection day even if the said number has already been achieved. This strategy is proposed to work towards attaining similar numbers of participants across all age groups to conduct subgroup analysis, if permitted. Any limitations arising from our research design and data observations will be reported along with research findings.

6. How will the under-age participants give their informed consent? Is the informed consent of parents/guardians required here?

Response: Considering our ethics approval, adolescents over 16 years were permitted to provide consent for their own participation. Furthermore, bearing in mind local ethics requirements in India, eligible participant were verbally informed about the project and were also given participant information sheet to take home and decide on their participation following discussions with their local guardian/parents. There was no financial incentive provided to avoid any possible coercion. No personal identifiable information was required or ever collected from participants where self-reported questionnaire strategy was used.

7. Survey questionnaire (Extended data): In my opinion, the wording of some items could be slightly changed in order to avoid misunderstandings and allow meaningful interpretation: Part A, item 5: In its current wording, the item might be understood as asking for the status quo of what "a women's role" usually is in society or what the adolescents experience in their daily life. Like this, their answer to the item is not necessarily reflective of a lack of support for gender inequality, as it does not directly ask whether the respondent agrees with this widespread societal role of women, or if they think it should be like that. If the item aims to assess the adolescents' gender beliefs and attitudes, I suggest rephrasing it slightly, for example like this: A woman's role should be taking care of her house and family members / A real/good woman's role is taking care of her house and family members. Similarly, in item 8 Part B, item 10: In this item to it is unclear whether it is asking for the adolescents' opinion/belief in this regard, or for their experience. This should be either rephrased or carefully considered in the interpretation of the data. 
Response: We prepared questions based on published literature on this subject, followed by discussions among team members, and then piloted involving adolescents from our study region. This process helped us identify issues around how certain questions were framed and those were revised. Further, advice on providing examples on some questions was implemented carefully. Nevertheless, we acknowledge that the survey tool could be further improved as you have explained. Due to the timeline of our project, data collection and analysis activities are now completed. However, we highly appreciate your advice, which is certainly very useful during future development of our survey tool. We have noted your guidance carefully to make further improvements and then aim to again pilot our survey tool in both rural and urban populations in India for future projects.

\section{Qualitative component:}

Data collection:

Given the sensitivity of the topic, the participants' gender identities should be considered. Will the focus groups consist of members of all genders or will they be separate for men and women members of the local gender promotion groups? Or are they all female anyway? And what about the gender of the research staff conducting the focus groups?

Response: This is indeed a very important area where project team discussed in detail during research design. All men and women work together through village level gender equality groups, and were also trained jointly as a team by the NGO partner over two years of period. Thus, all FGD will involve men and women during discussions. Two qualitative researchers collected these data having a combination of 1 male and 1 female researcher. As this was possible following a successful recruitment only, thus was not mentioned in this protocol. Nonetheless, we have now included following information to state this intention.

Focus group discussions will be conducted, transcribed, and translated by two experienced facilitators with bilingual skills (Marathi and English) under the supervision of the research team. Efforts will be made to recruit one male and one female researcher for this data collection task to achieve a gender balanced approach. This will provide a comfortable environment for all men and women members/study participants. Further, both researchers will lead on 6 FGD sessions each to provide equal opportunities for skill development through peer and supervisory feedback in line with our capacity development objectives.

\section{Conclusions:}

The authors state that "the project will provide opportunities to train and engage over 10 researchers and practitioners in Maharashtra state of India to improve their knowledge, develop new research skills, and enhance their experience of collaborating with international partners". From the described procedures, this expected outcome is not apparent. Please elaborate on what this assumption/aim is based on.

Response: We have revised this information and also linked with the project development and goal where capacity building initiative was mentioned. The revised conclusion is as follows.

The DEVELOP project will contribute to research capacity building and evidence-based practices in 
a resource limited setting to achieve our overarching project goal. The project will provide opportunities to train and engage a team of 12 local staff includes data collectors, assistants, researchers in the Maharashtra state of India to improve their knowledge, develop research skills, and enhance experiences of all institutes on international collaborations. It is expected that the project will help partners involved from India and the UK to continue research and also development work on the adoption, implementation, and scale-up of evidence-based gender equality interventions in Maharashtra and other Indian states and territories.

To the best of our knowledge, this will be the first survey from the Maharashtra state of India, and one of the largest surveys, measuring gender equality-related knowledge, attitudes, and behaviours among rural Indian adolescents. The survey findings will generate new valuable insights into how adolescent groups could be engaged in the future to improve gender equality in Indian communities.

The qualitative evaluation will inform the implementation of the 'Responsible Couples' intervention and strategies to improve the same through future expansion. It will also have policy implications for HMF, SWISSAID, and other organisations seeking to reduce DVA and empower women in Maharashtra and other parts of the country. Considering diverse Indian culture, practices, and beliefs, the study results should be interpreted carefully beyond the population studied.

\section{Minor corrections:}

10. Figure 1: the second lowest box on the right should read "Qualitative component"

Response: Thank you for noting this error. We have now requested to change this to ' Qualitative'. A figure indicating this change is also attached with the revised submission and submitted to the F1000.

Competing Interests: None to mention.

Reviewer Report 16 September 2019

https://doi.org/10.5256/f1000research.21401.r53221

(C) 2019 N Srinivas P. This is an open access peer review report distributed under the terms of the Creative Commons Attribution License, which permits unrestricted use, distribution, and reproduction in any medium, provided the original work is properly cited.

\section{Prashanth N Srinivas}

Institute of Public Health, Bengaluru, India

The paper is a study protocol of research proposed in Osmanabad district of Maharashtra, India to improve evidence-base for understanding and acting upon gender-based violence (GBV). The study seeks to understand gender equality related knowledge, attitudes and practices among adolescents on one hand, and evaluate an ongoing intervention to reduce gender-based violence, 
called "Responsible Couples" (RC). The authors make a strong case for the need for such evidence (from adolescents, where interventions targeting the problem ought to begin) as well as the relative lack of effectiveness evidence from interventions to reduce GBV. Authors indicate that this could be the first of its kind large survey on this topic in Maharashtra, and perhaps among Indian adolescents. In addition, their remarks on the research capacity-building and academic exchange involved are also noted with appreciation.

\section{Background}

The background provides a published estimate of domestic violence (DV) ever and DV in the past 6 months. The study cited surveyed households in one city of south India, whereas the background states this as being an estimate for south India, which may not be an appropriate representation of the original study's findings.

\section{Improving coherence through more contextual information and clarifying objectives} A project goal is separately provided in addition to the research objectives. The two research objectives focus on (1) survey tool development for adolescents, and (2) evaluation of the RC program. The project goal is quite broad and both objectives fit within this goal, but it is unclear how these two objectives come together. Is there any specific reason these two objectives have been chosen? What was the rationale for choosing this particular intervention? Perhaps this is related to specific contextual information that may not be part of the protocol. Researchers could consider a section that describes the particular setting where the study is being planned as well as perhaps a section that describes work leading up to the study to provide the reader an understanding of the logic/reasoning in choosing these two objectives in order to work towards the project goal.

While the background indicates that the authors will undertake a survey among adolescents (and indeed the data collection and subsequent activities confirm this, the first of the two objectives that include this appears to stop short at "...inform(ing) the development of a survey tool". This gives an impression of this being a tool-development/feasibility study only. Clarify.

\section{Selection and recruitment}

The project identifies 70 villages which correspond to the area of work of partner NGO as being the study area. It is unclear from the information provided how much of the district this covers, and what the original logic is for the partner NGO to choose these villages. Since a survey is being planned, the reporting of its findings will depend on the overall choice of study site and sampling and hence further information has to be provided to enable an understanding of the study area. Some information on the larger context in terms of how this district compares with other districts in the state with respect to GBV are useful (if available). An estimate of what proportion of the district is covered by these villages is also useful.

Authors indicate that the age of eligible respondents for the survey shall be 16 to 19 . How will this be verified (if at all)? Will this be based on self-reporting of age? Or will researchers have an estimate/list from prior work?

Will estimates/identity of adolescents in these villages be available to researchers from the existing work of partner NGO? If yes, specify how this data will be dealt with and used/not used. Authors may consider comparing their village-wise sample with adolescents in the 
village to come up with an estimate of their overall survey coverage among adolescents.

If adolescents who are unable to read/write turn up, what will the team do? There are both technical and ethical implications of this decision to exclude this group, even if the numbers in this group are small. More so given that educational status has been declared in the background to influence GBV.

\section{Sample size}

Authors state that one of the reasons for choosing a sample size of 1000 is "...to ensure that results from this study will be applicable to rural areas of Maharashtra state.". This will need to be explained clearly. Specifically, the concern is with respect to (a) how would any sample size defined within a set of villages in one district of the state "...be applicable" to the entire state? Perhaps the authors seek to achieve analytical generalisability of their findings through mixed-method/qualitative inquiry and demonstrate the relative similarity of few/many/all other districts to this district with respect to the area of inquiry, but this cannot be based on a particular sample size in my assessment. It is also noted that another logic to arrive at the size has been the resource availability. On the contrary, could an effort at achieving a sampling strategy of smaller numbers of household visits be used to further minimize sample numbers if needed? I understand that the work may have already begun, in which case, authors need to clarify this section appropriately.

Authors mention a stratified sampling approach, but the survey is being conducted by invitation to a "station" at each village. How will the 125 per each age-stratum and 500 from each gender be achieved if there is a patterning in age of children who turn up at the station (for instance, older boys being less "shy" of such a station, or for instance if some stations for reasons unknown are not acceptable/accessible for particular age/gender respondents). Given that the researchers do not identify specific strategies to ensure the sample across strata, this stratified sampling appears more to be a desire at ensuring distribution of sample across all age-groups rather than an actual sampling strategy? Clarify.

\section{RC program evaluation}

In terms of improving the coherence between these 2 objectives, and also given that both are being implemented in the same district, it is useful to understand if the 40 villages where RC will be evaluated are a sub-set of the 70 where the quantitative survey will be conducted?

The RC group in each village: Are these volunteers or paid staff of the project? Clarify.

Given that the objective is to evaluate an intervention which has multiple components, an overall evaluation approach to the intervention is missing. Partly, this could be dealt with by providing more details of how the qualitative data will be analyzed from the FGDs. Also to some extent, the evaluation will only engage with relative insiders (the village level RC group) and will not include perspectives of those who "received" the intervention, a limitation. Authors could consider including a clear schematic/narrative on how the intervention is expected to work outlining the various intervention inputs, assumptions being made and how it is linked to expected outputs of the intervention (akin to a theory of change). In the lack of such a theory, the qualitative data gathered may be difficult to coherently analyze. That said, if there is a plan for doing this in another way, authors could include that. 


\section{Data analysis}

There is limited information on how quantitative and qualitative data will be analyzed. This will have implications on the data collection methods and sampling details proposed. Also, see above about evaluation component and (lack of) details of the analysis proposed.

Is the rationale for, and objectives of, the study clearly described?

Partly

Is the study design appropriate for the research question?

Yes

Are sufficient details of the methods provided to allow replication by others? Partly

Are the datasets clearly presented in a useable and accessible format?

Not applicable

Competing Interests: No competing interests were disclosed.

Reviewer Expertise: Public health, realist evaluation, health policy \& systems research methods

I confirm that I have read this submission and believe that I have an appropriate level of expertise to confirm that it is of an acceptable scientific standard, however I have significant reservations, as outlined above.

Author Response 11 Mar 2021

Anand Ahankari, University of Hull, Hull, UK

Response to comments from reviewer 1 (Prashanth N Srinivas)

1. The paper is a study protocol of research proposed in Osmanabad district of Maharashtra, India to improve evidence-base for understanding and acting upon genderbased violence (GBV). The study seeks to understand gender equality related knowledge, attitudes and practices among adolescents on one hand, and evaluate an ongoing intervention to reduce gender-based violence, called "Responsible Couples" (RC). The authors make a strong case for the need for such evidence (from adolescents, where interventions targeting the problem ought to begin) as well as the relative lack of effectiveness evidence from interventions to reduce GBV. Authors indicate that this could be the first of its kind large survey on this topic in Maharashtra, and perhaps among Indian adolescents. In addition, their remarks on the research capacity-building and academic exchange involved are also noted with appreciation.

Response: Thank you for your careful reading of the manuscript and positive feedback. We believe your comments and suggestions have helped us to improve the quality of the manuscript. Please find below our point-by-point responses and revisions. 


\section{Background}

The background provides a published estimate of domestic violence (DV) ever and DV in the past 6 months. The study cited surveyed households in one city of south India, whereas the background states this as being an estimate for south India, which may not be an appropriate representation of the original study's findings.

Response: We have revised the introduction/background as follows.

A recent study involving women from marginalised groups in Bangalore city based in southern India reported that over 50\% of women had experienced physical domestic violence ever, and $27 \%$ faced physical violence in the past six months ${ }^{2}$.

\section{Improving coherence through more contextual information and clarifying objectives}

A project goal is separately provided in addition to the research objectives. The two research objectives focus on (1) survey tool development for adolescents, and (2) evaluation of the RC program. The project goal is quite broad and both objectives fit within this goal, but it is unclear how these two objectives come together. Is there any specific reason these two objectives have been chosen? What was the rationale for choosing this particular intervention? Perhaps this is related to specific contextual information that may not be part of the protocol. Researchers could consider a section that describes the particular setting where the study is being planned as well as perhaps a section that describes work leading up to the study to provide the reader an understanding of the logic/reasoning in choosing these two objectives in order to work towards the project goal.

Response: We have provided a more detailed explanation.

DEVELOP project is designed primarily to support new research collaborations by generating evidence mainly from rural areas of the Maharashtra state of India. The evidence generation is proposed by two key objectives which fit under the overarching project goal as explained below. Research work will involve local staff working full-time at HMF (NGO partner) who will be trained to develop their research capacities and will be engaged in data collection work. Research undertaken through both objectives by a local team will provide them 'real world' experiences supporting their future career development. Secondly, our two key partners (SWISSAID and HMF) will benefit from such evidence to inform their on-going as well as forthcoming projects on gender equality in India. The first objective will help our key partners plan projects involving young people through their future expansion, and the second objective will contribute to improve their on-going intervention approach. SWISSAID works with several Indian NGOs, thus findings will be useful in other areas of Maharashtra and nationally in India to design gender equality related work. Research findings, capacity building initiative and collaboration work will also offer valuable experiences for all partners involved to plan future initiatives. The proposed structure helped design the project in line with our funding requirements where local capacity building and research goals are incorporated into this model.

4. While the background indicates that the authors will undertake a survey among 
adolescents (and indeed the data collection and subsequent activities confirm this, the first of the two objectives that include this appears to stop short at "...inform(ing) the development of a survey tool". This gives an impression of this being a tooldevelopment/feasibility study only. Clarify.

Response: We have revised objectives to improve clarity and ensure that survey tool development and its proposed use is reflected with study location.

To develop a survey tool and measure gender equality related knowledge, attitudes, and behaviours in Indian adolescents using this tool in rural populations of the Maharashtra state of India.

To conduct a qualitative evaluation of the current DVA reduction intervention-the 'Responsible Couples' project implemented by HMF in rural areas of the Maharashtra state of India.

\section{Selection and recruitment}

The project identifies 70 villages which correspond to the area of work of partner NGO as being the study area. It is unclear from the information provided how much of the district this covers, and what the original logic is for the partner NGO to choose these villages. Since a survey is being planned, the reporting of its findings will depend on the overall choice of study site and sampling and hence further information has to be provided to enable an understanding of the study area. Some information on the larger context in terms of how this district compares with other districts in the state with respect to GBV are useful (if available). An estimate of what proportion of the district is covered by these villages is also useful.

Response: To the best of our knowledge, there are no published studies from this region to report, but we have provided information on the study area and connected this further with NGO's work.

The study area consists of 70 villages from HMF's project field, located in the Osmanabad district of Maharashtra state of India. HMF accelerated its programmes in this region following the 1993 earthquake to support emergency relief activities. This geographic area is known for limited healthcare facilities, poor infrastructure, and is also among marginalised regions nationally. Therefore, HMF's work focus has been in this region over 25 years where various welfare, education, health and development projects are regularly implemented. Majority of the population in the Osmanabad district is in rural areas (approximately $83 \%$ rural and $17 \%$ urban), and has about 733 villages in total across its 8 blocks. The proposed 70 villages are from two blocks namely Tuljapur and Lohara. The total district population is about 1.6 million and our study area has about 120-140,000 people across 70 villages.

6. Authors indicate that the age of eligible respondents for the survey shall be 16 to 19 . How will this be verified (if at all)? Will this be based on self-reporting of age? Or will researchers have an estimate/list from prior work?

Response: Data collection team will use self-reported age. All project staff were made aware about this requirement and such eligibility information is shared verbally as well as through 
participant information sheet.

The study will be open to both boys and girls aged 16 to 19 only. Information on this age related eligibility will be shared through staff trainings including field level support personnel who will ensure that this is shared correctly with potential eligible participants. Self-reported age will be verified verbally on the day of data collection by members of the local research team. Age requirement is mentioned on the participant information sheet which is provided in advance to eligible candidates (Supplementary 1, Extended data) ‥ Participants will be asked to report their age on the data collection form.

7. Will estimates/identity of adolescents in these villages be available to researchers from the existing work of partner NGO? If yes, specify how this data will be dealt with and used/not used. Authors may consider comparing their village-wise sample with adolescents in the village to come up with an estimate of their overall survey coverage among adolescents.

Response: Such information is not collected, stored or used anytime during the project due to the ethical implications. At the planning stage of the project, we considered estimating the overall survey coverage, but this was not feasible because unfortunately information on the numbers and socio-demographic characteristics of adolescents by village or region was not available.

8. If adolescents who are unable to read/write turn up, what will the team do? There are both technical and ethical implications of this decision to exclude this group, even if the numbers in this group are small. More so given that educational status has been declared in the background to influence GBV.

Response: Indeed, only those who are able to read and write will be able to participate in this study. This was preferred instead of interview questionnaire to ensure confidentiality and avoid reporting bias. From the experience of our partners and the lead researcher on our team who have previously conducted five research projects in this area, overall literacy skills among adolescents are sufficient to fully understand and participate in the survey. We have acknowledged this limitation in our revised draft.

We acknowledge that those who may not be able to read and write will not be eligible to participate in our study. However, considering our ethics processes and confidentiality needs to report gender equality related response with necessary privacy, self-reported approach is preferred.

\section{Sample size}

Authors state that one of the reasons for choosing a sample size of 1000 is "...to ensure that results from this study will be applicable to rural areas of Maharashtra state.". This will need to be explained clearly. Specifically, the concern is with respect to (a) how would any sample size defined within a set of villages in one district of the state "...be applicable" to the entire state? Perhaps the authors seek to achieve analytical generalisability of their findings through mixed-method/qualitative inquiry and demonstrate the relative similarity of few/many/all other districts to this district with respect to the area of inquiry, but this 
cannot be based on a particular sample size in my assessment. It is also noted that another logic to arrive at the size has been the resource availability. On the contrary, could an effort at achieving a sampling strategy of smaller numbers of household visits be used to further minimize sample numbers if needed? I understand that the work may have already begun, in which case, authors need to clarify this section appropriately. Authors mention a stratified sampling approach, but the survey is being conducted by invitation to a "station" at each village. How will the 125 per each age-stratum and 500 from each gender be achieved if there is a patterning in age of children who turn up at the station (for instance, older boys being less "shy" of such a station, or for instance if some stations for reasons unknown are not acceptable/accessible for particular age/gender respondents). Given that the researchers do not identify specific strategies to ensure the sample across strata, this stratified sampling appears more to be a desire at ensuring distribution of sample across all age-groups rather than an actual sampling strategy? Clarify.

Response: We have revised this section to improve clarity:

The adolescent survey will be conducted to collect a minimum of 1000 questionnaires. This number is based on discussions with project partners to ensure that study is deliverable in given resources and time. Research findings may be applicable to wider areas of rural Maharashtra state. The proposed target of 1000 is set considering three months will be available to collect data, however if permitted higher sample size will be achieved depending on project progress. $A$ similar strategy was used to conduct research in this field area involving adolescent girls $\underline{8}$. In order to collect representative data by age and gender, a stratified sampling effort will be undertaken. In total, a minimum 1000 questionnaires will be completed with an aim to collect 125 from each age group $(16,17,18,19$ years), and 500 from each gender. This is the ideal sample scenario; however, no participants will be turned away on a data collection day even if the said number has already been achieved. This strategy is proposed to work towards attaining similar numbers of participants across all age groups to conduct subgroup analysis, if permitted. Any limitations arising from our research design and data observations will be reported along with research findings.

\section{RC program evaluation}

In terms of improving the coherence between these 2 objectives, and also given that both are being implemented in the same district, it is useful to understand if the 40 villages where RC will be evaluated are a sub-set of the 70 where the quantitative survey will be conducted?

Response: Yes, the said 40 villages make the total of 70 . We have revised the manuscript accordingly.

The 'Responsible Couples' project is currently (in 2019) being implemented in 40 villages of Osmanabad district of Maharashtra state, India. These 40 villages are from the wider 70 village network outlined earlier where our NGO partner (HMF) is implementing development work. Each village has one local group comprised of 15 to 20 village members, who are voluntarily working towards gender equality in their community.

11. The RC group in each village: Are these volunteers or paid staff of the project? Clarify. 
Response: Village level gender equality group members work voluntarily. We have mentioned this in the revised text above.

12. Given that the objective is to evaluate an intervention which has multiple components, an overall evaluation approach to the intervention is missing. Partly, this could be dealt with by providing more details of how the qualitative data will be analyzed from the FGDs. Also to some extent, the evaluation will only engage with relative insiders (the village level RC group) and will not include perspectives of those who "received" the intervention, a limitation. Authors could consider including a clear schematic/narrative on how the intervention is expected to work outlining the various intervention inputs, assumptions being made and how it is linked to expected outputs of the intervention (akin to a theory of change). In the lack of such a theory, the qualitative data gathered may be difficult to coherently analyze. That said, if there is a plan for doing this in another way, authors could include that.

Authors response: We have secured a follow-up funding where qualitative interviews were conducted through the DEVELOP Phase 2 work. This involved in-depth interviews with men and women who utilised project services. Research data collection for this was completed in 2020, and currently this data are being prepared for analysis. We have mentioned this in our evaluation approach which is a new section included in the protocol.

\section{'Responsible Couples' project evaluation}

The proposed qualitative data collection through FGD with village level gender equality groups will contribute towards a full evaluation report. The proposed component is preferred during this initial stage of our work (DEVELOP Phase 1, 2018-19) to investigate community/village level change on gender equality related attitudes, behaviors to prevent and reduce DVA against women/girls. The future research (DEVELOP Phase 2, 2020-21) aims to conduct qualitative interviews with service users (men and women) who have used project services offered by village level groups and the NGO partner (HMF). For such future expansion, an independent funding will be sought. Qualitative data from both, service providers and users along with project monitoring reports by our partner organizations will generate evidence towards the final evaluation findings. We acknowledge that evaluating project services only from providers perspective will not be sufficient, thus future initiatives are planned, however such details are not included in this protocol considering it is beyond the current project's funding and timeframe (DEVELOP Phase 1, 2018-19).

\section{Data analysis}

There is limited information on how quantitative and qualitative data will be analyzed. This will have implications on the data collection methods and sampling details proposed. Also, see above about evaluation component and (lack of) details of the analysis proposed. Authors response: We have revised data analysis plan for both qualitative and quantitative sections as follows.

For quantitative component 
Incomplete questionnaires will be discarded from analysis and stored for auditing purposes. Details on such process including data validation steps will be provided in study methods/results. Survey responses will be analysed in Stata (StataCorp, College Station, Texas, USA) and/or SPSS (IBM) using descriptive statistics, tests of statistical significance, and reliability coefficients. Summary of all collected data will be presented through frequency and percentages for findings from the gender equality tool and research participant demographics. Cronbach's alpha score for the gender equality scale will be provided. The gender equality tool will be used to calculate a total score for each participant, and will be also used to report overall observations from on our study population. This score will be used as a continuous outcome of interest for linear regression purposes. In such analysis, data on individual sociodemographic parameters will be used as an independent exposure variables. Regression analysis will be adjusted depending on availability of data, statistical guidance and published evidence. If data permit, then additional analysis such as logistic regression will be conducted along with comparing results across villages/blocks. Results will be reported in line with STROBE guidelines 11 , and will be submitted for a peer review publication.

For qualitative component

Qualitative data from the focus group discussions will be analysed thematically, where two researchers will independently code data and synthesise findings into themes. This will be informed by Braun and Clark's deductive reasoning methodology 12 . Additional inputs will be provided by senior qualitative researchers to supervise this process and will also contribute towards such analysis. Furthermore, researchers working on this data will then meet to discuss areas of agreement and disagreement and reach consensus on the coding tree, illustrative quotations, and interpretation. All data findings will be shared with key project partners and data collection to conduct internal peer reviews and checks prior to finalising themes and key findings. Presentation guidelines such as COREQ will be followed wherever deemed necessary 13 . All agreed themes and sub-themes will be reported in the study results.

Competing Interests: None to mention 
The benefits of publishing with F1000Research:

- Your article is published within days, with no editorial bias

- You can publish traditional articles, null/negative results, case reports, data notes and more

- The peer review process is transparent and collaborative

- Your article is indexed in PubMed after passing peer review

- Dedicated customer support at every stage

For pre-submission enquiries, contact research@f1000.com 